\title{
ESTRUTURA DO SOLO SOB FEIJÃO IRRIGADO E DIFERENTES MANEJO DO SOLO
}

\author{
Lorena Adriana de Gennaro(1), Zigomar Menezes de Souza ${ }^{(1)}$, Laura Fernanda Simões da \\ Silva $^{(1)}$, Miguel Cooper ${ }^{(2)}$ e Milton César Costa $\operatorname{Campos}^{(3)^{*}}$ \\ (1) Universidade Estadual de Campinas, Faculdade de Engenharia Agrícola, Departamento de Água e Solos, Campinas, São Paulo, \\ Brasil. \\ (2) Universidade de São Paulo, Escola Superior de Agricultura Luiz de Queiroz, Departamento de Ciência do Solo, Piracicaba, São \\ Paulo, Brasil. \\ (3) Universidade Federal do Amazonas, Instituto de Educação, Agricultura e Ambiente, Humaitá, Amazonas, Brasil. \\ * Autor correspondente. \\ E-mail: mcesarsolos@gmail.com
}

\section{RESUMO}

O manejo agrícola influencia a estrutura do solo, modificando os atributos físicos e o comportamento hídrico do solo. $O$ objetivo deste trabalho foi avaliar os efeitos da semeadura direta (SD) e do preparo convencional (PC) sobre a estrutura do solo, por meio de atributos físicos e da distribuição dos poros, utilizando imagens digitais 2-D. O estudo foi realizado em um Latossolo Vermelho distroférrico com delineamento de blocos casualizados. A densidade do solo, macroporosidade, diâmetro médio ponderado (DMP), condutividade hidráulica do solo saturado (Kfs) e análise de imagens digitais 2-D (resolução de 156,25 $\mathrm{\mu m}^{2}$ ) foram avaliadas nas profundidades de 0-0,20 e 0,20-0,40 $\mathrm{m}$. Os manejos não diferiram para a densidade e macroporosidade do solo. A SD revelou maior DMP e Kfs em relação ao PC. O PC apresentou maior área total de poros na profundidade de 0-0,20 $\mathrm{m}$ em relação ao $\mathrm{SD}$, ocorrendo o inverso na profundidade de $0,20-0,40 \mathrm{~m}$. A SD apresentou maior quantidade de poros complexos de tamanho grande $\left(>0,156 \mathrm{~mm}^{2}\right)$ na camada de 0,20-0,40 $\mathrm{m}$ em relação ao $\mathrm{PC}$, que evidenciou predomínio de poros arredondados. A SD demonstrou melhorias na estrutura do solo em relação ao PC, com maior estabilidade dos agregados, condutividade hidráulica e área total de poros, contribuindo para a conservação do solo e da água em sistemas produtivos.

Palavras-chave: preparo convencional, semeadura direta, análise de imagem, monólitos, Phaseolus vulgaris. 


\title{
ABSTRACT: SOIL STRUCTURE UNDER IRRIGATED BEANS AND DIFFERENT SOIL MANAGEMENT PRACTICES
}

\begin{abstract}
Agricultural management affects soil structure, modifying soil physical properties and soil water movement. The objective of this study was to evaluate the effects of no-tillage (NT) and conventional tillage $(C T)$ on soil structure through physical properties and pore distribution in 2-D digital images. The study was conducted in an Oxisol with a randomized block design. Soil bulk density, soil macroporosity, mean weighted diameter (MWD), saturated hydraulic conductivity (SHC), and analysis of 2-D digital images (156.25 $\mathrm{\mu m}^{2}$ resolution) were evaluated at the depths of 0-0.20 and 0.20-0.40 m. Management systems did not differ for soil bulk density and soil macroporosity. The NT showed higher MWD and SHC compared to CT. The CT had a greater total pore area at the $0-0.20 \mathrm{~m}$ depth in relation to NT, with the opposite occurring at the 0.20-0.40 $\mathrm{m}$ depth. The NT had a greater number of complex pores of large size $\left(>0.156 \mathrm{~mm}^{2}\right)$ at the depth of $0.20-0.40 \mathrm{~m}$ in relation to the CT, which showed a predominance of rounded pores. The NT showed improvements in soil structure in relation to the CT, with greater aggregate stability, hydraulic conductivity, and total pore area, contributing to soil and water conservation in production systems.
\end{abstract}

Keywords: conventional tillage, no-tillage, image analysis, monoliths, Phaseolus vulgaris.

\section{INTRODUÇÃO}

O uso sucessivo do preparo convencional do solo sem um adequado plano de rotação de culturas e uma manutenção da cobertura morta sobre o solo em áreas de produção agrícola modifica os seus atributos físicos (Pagliai et al., 2004; Salton et al., 2008; Rossetti e Centurion, 2013). As principais alterações ocorrem na estrutura do solo associadas à formação de camadas compactadas com diminuição do volume de macroporos, tamanho de agregados, taxa de infiltração de água e aumento da sua resistência do solo à penetração e densidade (Souza et al., 2006; Hou et al., 2012; Souza et al., 2014). As operações mecanizadas sobre o solo proporcionam ainda alterações na forma dos poros, com redução do diâmetro e na continuidade dos canais, o que diminui a permeabilidade da água (Lima et al., 2005; Pagliai e Kutilek, 2008; Castro et al., 2009). Dessa forma, a redução no preparo sucessivo do solo associada à semeadura direta e ao acúmulo de resíduos orgânicos na sua superfície podem minimizar ou reverter o seu depauperamento, contribuindo para a sua conservação e da água nos agroecossistemas (Mallory et al., 2011; Rossetti e Centurion, 2013; Moreira et al., 2014).

O impacto da compactação na estrutura do solo pode ser analisado com o estudo micromorfológico, que utiliza análise de imagens digitalizadas 2-D, que geram índices quantitativos quanto à forma e complexidade dos poros (Souza et al., 2006; Pires et al., 2009; Cooper et al., 2010). Assim, a estrutura do solo pode ser representada por tamanho, forma e arranjo das partículas primárias, com materiais agregados ou não, e seu respectivo espaço poroso (Castro et al., 2003; Cooper et al., 2010, 2012). A análise da estrutura do solo possibilita verificar as alterações impostas pelo sistema de preparo e o manejo na condução da cultura. Porém, para o estudo detalhado de determinadas modificações, em escala milimétrica ou micrométrica, têm sido utilizados aparelhos microscópicos e lâminas delgadas, para melhor visualização dos constituintes estruturais do solo (Castro et al., 2003; Pagliai e Kutilek, 2008; Cooper et al., 2012).

A análise da porosidade e estrutura do solo, por meio de imagens digitalizadas, tem sido usada como técnica eficiente e útil para observar as alterações que o manejo acarreta na microestrutura do solo, influenciando também na macroestrutura (Castro et al., 2003; Souza et al., 2006; Pires et al., 2009; Cooper et al., 2010). É uma ferramenta que permite observar a estrutura do solo na sua forma natural, possibilitando melhor visualização no comportamento da estrutura e do espaço poroso em diferentes sistemas de manejo ou em áreas sob processo de degradação (Juhász et al., 2007; Pagliai e Kutilek, 2008; Pires et al., 2009; Cooper et al., 2012).

O uso de técnicas de análise de imagens para estudos quantitativos e qualitativos da estrutura do solo tornou-se mais comum na literatura com o advento de equipamentos e programas (softwares) mais acessíveis (Souza et al., 2006; Juhász et al., 2007; Pires et al., 2009; Cooper et al., 2010). Essas técnicas, associadas à micromorfologia, permitem o estudo quantitativo de modificações estruturais em amostras indeformadas, após seu preparo e sua montagem em lâminas ou blocos polidos. Vários estudos por meio de técnicas micromorfológicas têm demonstrado que o cultivo altera a porosidade do solo, promovendo modificações nos atributos físicos (Fox et al., 2004; Souza et al., 2006; Cooper et al., 2012; Elyeznasni et al., 2012).

O uso de técnicas micromorfológicas representa nova visão para diagnósticos precisos de alterações na estrutura do solo, bem como de seus atributos 
físicos diretamente relacionados com a sua qualidade física. Portanto, o objetivo deste trabalho foi avaliar os efeitos da semeadura direta (SD) e do preparo convencional (PC) sobre a estrutura do solo, por meio de atributos físicos e da distribuição dos poros, utilizando imagens digitais 2-D.

\section{MATERIAL E MÉTODOS}

O experimento foi instalado em blocos inteiramente casualizados, com quatro repetições, em uma área de Latossolo Vermelho distroférrico típico (Embrapa, 2013), altitude média de $640 \mathrm{~m}$ e coordenadas geográficas de $22^{\circ} 48^{\prime} 57^{\prime \prime}$ de latitude sul e $47^{\circ} 03^{\prime} 33^{\prime \prime}$ de longitude oeste. $\mathrm{O}$ clima regional pela classificação de Köppen corresponde a uma transição entre os tipos Cwa e Cfa, indicativo de clima tropical de altitude com inverno seco e verão úmido. A precipitação pluvial média anual é de $1.382 \mathrm{~mm}$, com o período chuvoso entre outubro e março (Gennaro et al., 2014).

Os manejos avaliados foram: preparo convencional (PC), com uma passada da grade aradora para o revolvimento do solo a $0,10 \mathrm{~m}$ de profundidade; e semeadura direta (SD) sobre restos culturais de aveia-preta. $\mathrm{O}$ experimento foi montado na safra de 2003/04, com as avaliações realizadas na safra de 2007/08. Parcelas experimentais de $30 \times 20 \mathrm{~m}$ foram usadas com rotação de milho, feijão e aveia-preta, intercalados com períodos em pousio nos plantios de verão e inverno.

A cultura instalada no período do experimento foi o feijoeiro variedade IAC-Carioca. O espaçamento da cultura foi de $0,45 \mathrm{~m}$ entrelinhas, com 12 sementes por metro linear e população de 230.000 plantas ha-1. A emergência das plântulas ocorreu oito dias após a semeadura. A adubação básica nos sulcos de semeadura foi realizada levando-se em consideração a análise de solo, aplicando $200 \mathrm{~kg} \mathrm{ha}^{-1}$ da fórmula 4-14-8. A adubação nitrogenada em cobertura foi realizada aos 25 dias após a emergência, quando as plantas encontravam-se no estádio V4 (emissão da terceira folha trifoliada), aplicando $20 \mathrm{~kg} \mathrm{ha}^{-1}$ de $\mathrm{N}$ na forma de ureia. Nas safras de inverno, foi realizada irrigação por aspersão convencional, mantendo o teor de água do solo próximo à capacidade de campo, aplicando uma lâmina total de irrigação de $285,8 \mathrm{~mm}$.

Após a colheita do feijão, amostras indeformadas de solo $(n=80)$ foram coletadas em cilindros volumétricos de $0,05 \times 0,05 \mathrm{~m}$ nas profundidades de 0-0,20 e 0,20-0,40 m em trincheiras distribuídas aleatoriamente nos dois manejos para determinar a densidade do solo e macroporosidade, após saturação por capilaridade e aplicando tensão de $6 \mathrm{kPa}$ (Embrapa, 2011). Amostras com estrutura preservada $(n=80)$ foram coletadas para análise da estabilidade de agregados, realizada por tamisamento em via úmida (Kemper e Chepil,
1965), utilizando cinco peneiras com diâmetros de 4,$76 ; 2,0 ; 1,0 ; 0,5$; e $0,25 \mathrm{~mm}$, para obter as classes de agregados. A estabilidade de agregados foi representada pelo diâmetro médio ponderado dos agregados (DMP), também realizada por Souza et al. (2014). Na avaliação em campo da condutividade hidráulica do solo saturado (Kfs) ( $\mathrm{n}=80$ ), empregou-se o permeâmetro de Guelph, modificado por Vieira (1998). Mediu-se a taxa constante de infiltração de água no solo e calculou-se a Kfs, de acordo com Reynolds e Elrick (1985).

A área dos poros do solo foi determinada por meio da análise de imagens digitalizadas 2-D em amostras de solo de estrutura preservada. Monólitos de solo $(\mathrm{n}=16)$ com dimensões de 0,07 $\times 0,12 \times 0,06 \mathrm{~m}$ foram coletados nos dois manejos nas profundidades 0-0,20 e 0,20-0,40 m. Os monólitos foram secos e, posteriormente, impregnados por capilaridade em câmara de vácuo com resina hidrofóbica de poliéster com monômero de estireno, adicionada a um pigmento fluorescente Tinopal OB $\left(\mathrm{BASP}^{\circledR}\right)$. Após impregnação os monólitos foram cortados e polidos.

Para quantificar a porosidade, os blocos polidos foram iluminados com luz ultravioleta e fotografados com o auxílio de uma câmera digital (Sony ${ }^{\mathbb{B}}$, modelo DFW-X700), em sistema Charged Couple Device (CCD), acoplada ao microscópio petrográfico. Para cada bloco, foram obtidas aleatoriamente 15 microfotografias, compondo imagens $(\mathrm{n}=240)$ com dimensões de $0,012 \times 0,015 \mathrm{~m}$. As imagens foram digitalizadas com resolução espacial de $1024 \times 768$ pixels, com resolução espectral de 256 tons de cinza, em aumento de 10 vezes; cada pixel corresponde a uma área de 156,25 $\mathrm{\mu m}^{2}$.

A identificação dos poros na imagem foi feita pela delimitação da limiarização (thresholding) de tons de cinza para cada conjunto de imagens e, em seguida, realizada a etapa de binarização, empregando o programa Noesis Visilog $5.4^{\circledR}$, conforme Cooper et al. (2010). A porosidade total (PT) do solo foi calculada pela relação percentual entre a soma das áreas dos poros e a área total do campo imageado. Os poros foram divididos de acordo com sua forma em três grupos: arredondados, alongados e complexos; e quanto ao tamanho em: pequeno $\left(0,00156-0,0156 \mathrm{~mm}^{2}\right)$, médio $\left(0,0156-0,156 \mathrm{~mm}^{2}\right)$ e grande $\left(>0,156 \mathrm{~mm}^{2}\right)$, conforme Cooper et al. (2010). Dois índices (I1 e I2) foram utilizados para determinar a forma dos poros (Quadro 1).

$$
\begin{array}{ll}
I_{1}=\frac{p^{2}}{(4 \pi \mathrm{A})} & \text { Eq. } 1 \\
I_{2}=\frac{\frac{1}{m} \sum_{i}\left(N_{I}\right) i}{\frac{1}{n} \sum_{j}\left(N_{F}\right) i} & \text { Eq. } 2
\end{array}
$$

em que $P$ é o perímetro do poro, $A$ é a área do poro, $N_{I}$ é o número de interceptos de um objeto na direção i (i $=0^{\circ}, 45^{\circ}, 90^{\circ}$ e $\left.135^{\circ}\right), D_{F}$ é o diâmetro de Feret de um objeto na direção $\mathrm{j}\left(\mathrm{j}=0^{\circ}\right.$ e $\left.90^{\circ}\right), m$ e $n$ 
são o número de $i$ e $j$ direções, respectivamente. $I_{1}$ é igual a um para uma forma perfeitamente circular e é maior à medida que a forma desvia-se do círculo. $I_{2}$ complementa $I_{1}$ para obter uma precisão na separação entre alongados e complexos.

Os resultados dos atributos do solo foram submetidos à análise de variância considerando a significância $(p>0,05)$ para o teste $F$ e as médias foram comparadas pelo teste de Tukey $(p>0,05)$, utilizando-se o software Statistical Analysis System (SAS).

\section{RESULTADOS E DISCUSSÕES}

Os manejos SD e PC não diferiram para a densidade e macroporosidade do solo nas duas profundidades em estudo (Quadro 2). Era esperado diferença entre os manejos, contudo essa similaridade entre SD e PC pode estar associada à não consolidação dos manejos, estando em processo de transformação. Assis e Lanças (2005), estudando um Nitossolo Vermelho distroférrico em Botucatu, SP, verificaram efeito positivo da SD após 12 anos de implantação do manejo para a densidade e macroporosidade do solo. Vale ressaltar, entretanto, que o SD apresentou valores de macroporosidade do solo superiores a $0,10 \mathrm{~m}^{3} \mathrm{~m}^{-3}$ para as profundidades de $0,00-0,20 \mathrm{e}$ 0,20-0,40 m, o que tem sido considerado um valor mínimo adequado para as trocas gasosas entre o ambiente externo e o solo (Rossetti e Centurion, 2013). Dessa forma, valores menores, como os

Quadro 1. Critérios para distinção entre grupos quanto à forma dos poros

\begin{tabular}{lcc}
\hline \multirow{2}{*}{ Poro } & \multicolumn{2}{c}{ Índice de forma } \\
\cline { 2 - 3 } & I1 & I2 \\
\hline Arredondados & $\mathrm{I} 1 \leq 5$ & - \\
Alongados & $5<\mathrm{I} 1 \leq 25$ & $\mathrm{I} 2 \leq 2,2$ \\
Complexos & $5<\mathrm{I} 1 \leq 25$ ou $\mathrm{I} 1>25$ & $\mathrm{I} 2>2,2$ \\
\hline
\end{tabular}

observados no PC, são considerados limitantes ao crescimento das raízes de culturas agrícolas (Pagliai et al., 2004; Reichert et al., 2007; Souza et al., 2014). Esses resultados concordaram com os de Rossetti e Centurion (2013), os quais também observaram macroporosidade inferior a $0,10 \mathrm{~m}^{3} \mathrm{~m}^{-3}$ em um Latossolo Vermelho na camada superficial em SD com oito anos de implantação.

Os agregados do solo estáveis em água sob SD apresentaram os maiores valores para o diâmetro médio ponderado (DMP), em relação ao $\mathrm{PC}$ nas duas profundidades em estudo (Quadro 2). Esses resultados pactuaram com os de Salton et al. (2008) e Hickmann e Costa (2012), comparando o sistema plantio direto ao preparo convencional do solo. A maior estabilidade de agregados em sistemas conservacionistas, como a SD na palha, deve-se, entre outros fatores, ao contínuo fornecimento de material orgânico, que serve como fonte de energia para a atividade microbiana, que atua como agente na estabilização dos agregados (Salton et al., 2008; Hou et al., 2012; Souza et al., 2014).

No PC, o revolvimento do solo antes do plantio aumenta a decomposição da matéria orgânica do solo, refletindo em seu decréscimo ao longo dos anos e, consequentemente, diminuindo a estabilidade de agregados, o que está de acordo com Hickmann e Costa (2012). No preparo convencional do solo ocorre o tráfego de máquinas com efeito acumulativo das passadas dos pneus ao longo dos anos de cultivo, que resulta na compressão das partículas do solo, sem, entretanto, ocorrer os mecanismos que contribuem para a estabilização dos torrões, não apresentando as qualidades positivas ao desenvolvimento radicular de um agregado (Hickmann e Costa, 2012; Hou et al., 2012). Esse tipo de agregado possui menor grau de resistência interna, o que causa a deterioração da estrutura do solo, formando agregados grandes, porém, densos e fracos, com poucos poros interagregados e de menor diâmetro, criando um ambiente com maiores restrições ao desenvolvimento radicular (Williams e Weill, 2004).

$\mathrm{Na}$ SD e no PC, foram observadas redução do DMP em profundidade (Quadro 2), o que está relacionada

Quadro 2. Densidade do solo (DS), macroporosidade (Macro), diâmetro médio ponderado (DMP) e condutividade hidráulica (CH) em duas profundidades de um Latossolo Vermelho distroférrico típico manejado com semeadura direta (SD) ou preparo convencional (PC)

\begin{tabular}{|c|c|c|c|c|c|c|c|c|}
\hline \multirow[t]{3}{*}{ Manejo } & \multicolumn{8}{|c|}{ Profundidade (m) } \\
\hline & $0,00-0,20$ & $0,20-0,40$ & $0,00-0,20$ & $0,20-0,40$ & $0,00-0,20$ & $0,20-0,40$ & $0,00-0,20$ & $0,20-0,40$ \\
\hline & \multicolumn{2}{|c|}{ DS } & \multicolumn{2}{|c|}{ Macro } & \multicolumn{2}{|c|}{ DMP } & \multicolumn{2}{|c|}{$\mathbf{C H}$} \\
\hline & \multicolumn{2}{|c|}{$\mathrm{kg} \mathrm{dm}^{-3}$} & \multicolumn{2}{|c|}{$\mathrm{m}^{3} \mathrm{~m}^{-3}$} & \multicolumn{2}{|c|}{$\mathrm{mm}$} & \multicolumn{2}{|c|}{$\mathrm{mm} \mathrm{h}^{-1}$} \\
\hline SD & $1,32 \mathrm{a}$ & $1,28 \mathrm{a}$ & $0,10 \mathrm{a}$ & $0,13 \mathrm{a}$ & $2,60 \mathrm{a}$ & $1,95 \mathrm{a}$ & $112,00 \mathrm{a}$ & $99,10 \mathrm{a}$ \\
\hline $\mathrm{PC}$ & $1,28 \mathrm{a}$ & $1,25 \mathrm{a}$ & $0,07 \mathrm{a}$ & $0,09 \mathrm{a}$ & $1,95 \mathrm{~b}$ & $1,42 \mathrm{~b}$ & $91,30 \mathrm{~b}$ & $82,21 \mathrm{~b}$ \\
\hline $\mathrm{CV}(\%)$ & 3,70 & 4,60 & 4,82 & 3,51 & 15,41 & 13,21 & 45,62 & 53,32 \\
\hline
\end{tabular}

Médias seguidas por mesma letra maiúscula na coluna não diferem entre si pelo teste de Tukey (p>0,05). CV: coeficiente de variação. 
com a diminuição do teor de matéria orgânica do solo em profundidade (dados não apresentados), conforme observado em Mallory et al. (2011) e Gennaro et al. (2014). A matéria orgânica apresenta grande superfície específica e capacidade de troca de cátions, possibilitando maior número de ligações eletrostáticas com as partículas de solo, conferindo maior estabilidade aos agregados (Hickmann e Costa, 2012). Além disso, a sua decomposição por microrganismos resulta na formação de inúmeros compostos importantes na cimentação e estabilização dos agregados (Salton et al., 2008). Esses resultados também concordaram com os de Souza et al. (2014), os quais também observaram redução do DMP em profundidade em um Latossolo Vermelho sob diferentes sistemas de manejo.

A condutividade hidráulica do solo saturado (Kfs) variou de 99,10 a 112,00 e de 82,21 a $91,30 \mathrm{~mm} \mathrm{~h}^{-1}$ para SD e PC, respectivamente (Quadro 2). Assis e Lanças (2005), avaliando os atributos físicos em um Nitossolo Vermelho, verificaram condutividade hidráulica saturada variando de 127 a $254 \mathrm{~mm} \mathrm{~h}^{-1}$ para a SD com 12 anos de implantação. Menores valores de Kfs foram observados em PC em relação à SD nas duas profundidades, causada pela desestruturação do solo na camada arável e compactação do solo em subsuperfície ("pé de grade"), em decorrência do preparo periódico do solo, resultando em maior quantidade de partículas finas em suspensão, o que favorece o selamento superficial e o entupimento de poros. A maior Kfs na SD indica maior continuidade dos poros, que facilita a movimentação tridimensional da água (Assis e Lanças, 2005). Resultados semelhantes foram observados por Castro et al. (2009), verificando que a SD permite maior taxa de infiltração e condutividade hidráulica do solo saturado em superfície e em profundidade, quando comparada com os sistemas de preparo mecânico. A Kfs apresentou maiores valores na camada de 0,00-0,20 em relação a 0,20-0,40 m nos dois sistemas analisados, o que está relacionado à região de maior crescimento radicular das culturas agrícolas, com as raízes criando microtúbulos após a morte e decomposição do tecido vegetal, que favorecem o movimento de água no solo (Williams e Weill, 2004). Esses resultados acordaram com os de Castro et al. (2009) e Cooper et al. (2012).

A análise de imagens digitalizadas 2-D indicou alterações na porosidade total (PT) causadas pelos manejos do solo (Figura 1). O PC apresentou maior PT na camada de 0,00-0,20 $\mathrm{m}$ em relação à SD (Figuras 1a e 1c), ocorrendo o inverso na camada de 0,20-0,40 m (Figuras 1b e 1d), indicando o efeito superficial do preparo periódico do solo, contudo ocorrendo a compactação do solo em subsuperfície realizada pelos órgãos ativos da grade aradora durante o preparo do solo. A SD apresentou aumento da PT em profundidade (Figuras 1a e 1b), o que é associado ao efeito cumulativo do tráfego de máquinas na camada superficial e à melhoria da estrutura do solo em subsuperfície. No PC ocorreu a redução da PT em profundidade (Figuras 1c e 1d), proporcionada pelo revolvimento da camada arável e pela compactação em subsuperfície ("pé de grade"). Esses resultados concordaram com os de Lima et al. (2005), os quais, estudando diferenças entre sistemas de manejo por meio de análise de imagens digitalizadas 2-D e micromorfologia, observaram alterações na porosidade do solo provocado pelo tráfego de máquinas agrícolas. Moreira et al. (2014) também observaram na SD aumento da densidade e redução da macroporosidade do solo na camada superficial.

A SD apresentou predomínio de poros complexos de tamanho grande $\left(>0,156 \mathrm{~mm}^{2}\right)$ na profundidade de 0,00-0,20 e 0,20-0,40 m (Figuras 1a e 1b), indicando melhor estruturação do solo, com poros interconectados, promovida pelo aporte de restos vegetais e desenvolvimento radicular de diferentes culturas. Esses resultados acordaram com os de Salton et al. (2008) e Williams e Weill (2004). Os poros complexos promoveram maior condutividade hidráulica (Quadro 2), concordando com os resultados observados por Pagliai e Kutilek (2008) e Cooper et al. (2012). No PC, predominaram-se poros complexos e grandes na superfície, mas arredondados e médios $\left(0,0156-0,156 \mathrm{~mm}^{2}\right)$ em subsuperfície (Figuras 1c e 1d). Esses resultados representaram o efeito da gradagem sobre a camada arável, com a compactação do solo em subsuperfície, causado por sucessivos preparos do solo numa mesma profundidade. A maior proporção de poros arredondados no PC em subsuperfície é responsável por menor condução de água ao longo do perfil do solo em razão de menor conectividade entre poros (Quadro 2). Esses resultados concordaram com os de Souza et al. (2006), os quais, estudando a micromorfologia do solo e sua relação com atributos físicos e hídricos, verificaram menor condutividade hidráulica do solo. Maior movimento de água ao longo do perfil por meio dos poros complexos também foram observados por Fox et al. (2004) e Elyeznasni et al. (2012).

A SD promoveu, em média, maior formação de poros complexos no solo, responsáveis por maior condutividade hidráulica do solo (Figuras 1a e 1b, Quadro 2). Esses poros complexos são formados principalmente por processos biológicos, basicamente o enraizamento das culturas utilizadas na rotação, atuando como indicativo da degradação estrutural do solo (Williams e Weill, 2004; Lima et al., 2005; Salton et al., 2008). Assim, a SD condicionou a melhoria da estrutura do solo, com maior variação de formas e tamanhos de poros, que funcionam como rotas alternativas para o crescimento das raízes e favorecem a infiltração de água no solo (Williams e Weill, 2004; Pagliai e Kutilek, 2008). Esses poros complexos reduzem as limitações físicas ao crescimento das raízes (Souza et al., 2006), o que pode aumentar a produtividade da cultura (Williams e Weill, 2004). 

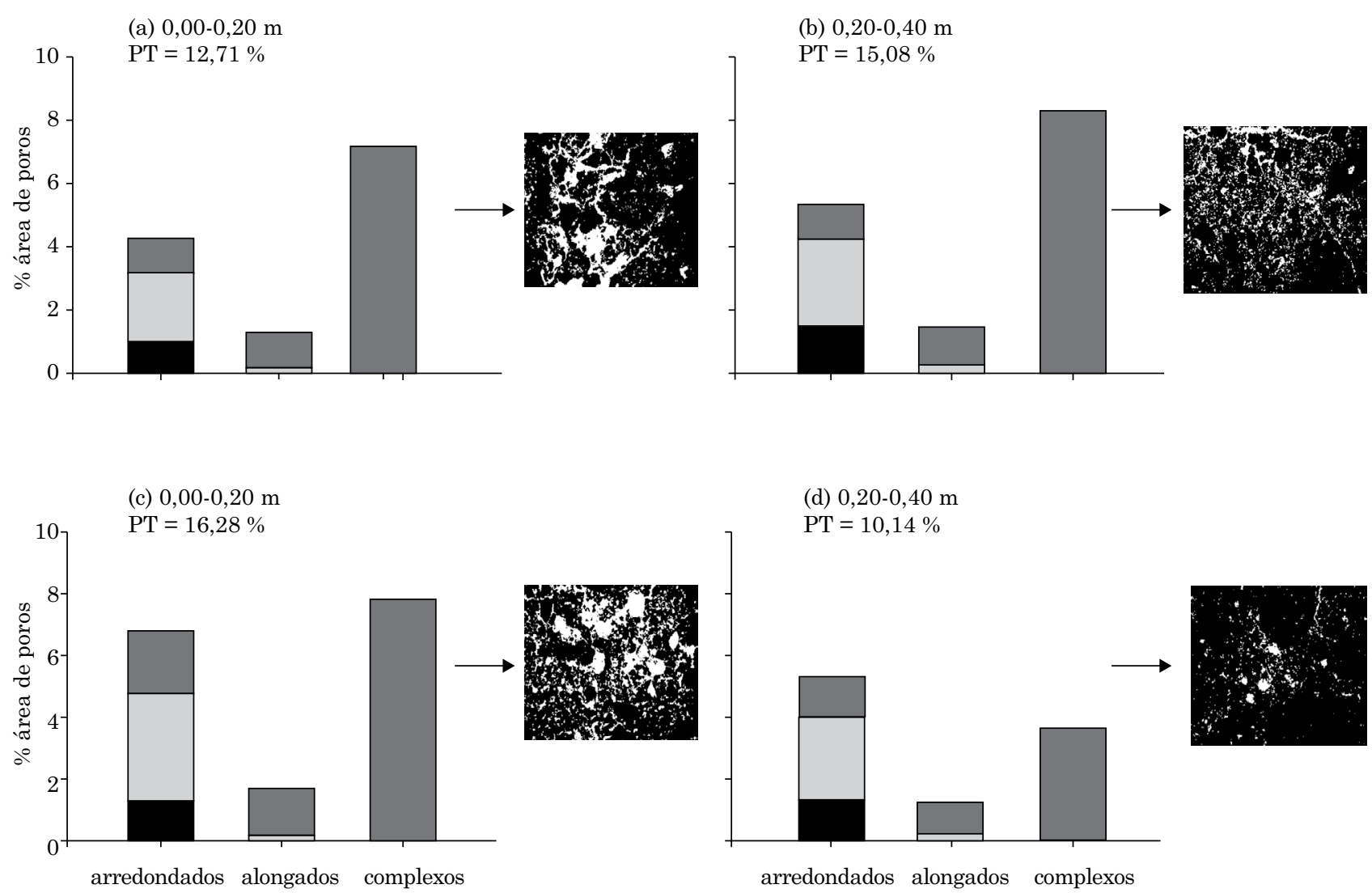

Figura 1. Forma e tamanho de poros quantificados pelas imagens 2D (poros em branco, fase sólida em preto) e porosidade total (PT) em duas profundidades de um Latossolo Vermelho distroférrico típico manejado com semeadura direta [SD: (a), (b)] ou preparo convencional [PC: (c), (d)]. Poros: $m$ Pequeno $\left(0,00156-0,0156 \mathrm{~mm}^{2}\right)$; Médio $\left(0,0156-0,156 \mathrm{~mm}^{2}\right)$; e Grande $\left(>0,156 \mathrm{~mm}^{2}\right)$. (a) SD 0,00-0,20 m; (b) SD 0,20-0,40 m; (c) PC 0,00-0,20 m; e (d) PC 0,20-0,40 m.

\section{CONCLUSÕES}

A semeadura direta apresentou melhorias na estrutura do solo em relação ao plantio convencional nas duas camadas em estudo, com maior estabilidade dos agregados, condutividade hidráulica e área total de poros, com predomínio de poros complexos e grandes, contribuindo para a conservação do solo e da água nos sistemas produtivos.

A análise de imagens digitalizadas 2-D foi mais sensível que a densidade e a macroporosidade do solo para quantificar alterações causadas pelo manejo, pelo maior detalhamento dos poros quanto à quantidade, ao tamanho e à forma.

\section{AGRADECIMENTOS}

Ao $\mathrm{CNPq}$, pela bolsa concedida ao primeiro autor; e à FAPESP, pelo financiamento do projeto (Processo: 2006/07072-3).

\section{REFERÊNCIAS}

Assis RL, Lanças KP. Avaliação dos atributos físicos de um Nitossolo Vermelho distroférrico sob sistema plantio direto, preparo convencional e mata nativa. $\mathrm{R}$ Bras Ci Solo. 2005;29:515-22.

Castro OM, Vieira SR, Siqueira GM, Andrade CA. Atributos físicos e químicos de um Latossolo Vermelho eutroférrico sob diferentes sistemas de preparo. Bragantia. 2009;68:1047-57.

Castro SS, Cooper M, Santos MC, Vidal-Torrado P. Micromorfologia do solo: bases e aplicações. In: Curi N, Marques JJ, Guilerme LRG, Lima JM, Lopes AS, Alvarez V. VH, editores. Tópicos em ciência do solo. Viçosa, MG: Sociedade Brasileira de Ciência do Solo; 2003. v.3, p.107-64.

Cooper M, Dalla Rosa J, Medeiros JC, Oliveira TC, Toma RS, Juhász CEP. Hydro-physical characterization of soils under tropical semi-deciduous forest. Sci Agric. 2012:69:152-9.

Cooper M, Vidal-Torrado P, Grimaldi M. Soil structure transformations from ferralic to nitic horizons on a toposequence in southeastern Brazil. R Bras Ci Solo. 2010:34:1685-99. 
Elyeznasni N, Sellami F, Pot V, Benoit P, Vieublé-Gonod L, Young I, Peth S. Exploration of soil micromorphology to identify coarse-sized OM assemblages in X-ray CT images of undisturbed cultivated soil cores. Geoderma. 2012;179/180:38-45.

Empresa Brasileira de Pesquisa Agropecuária - Embrapa. Centro Nacional de Pesquisa de Solos. Sistema brasileiro de classificação de solos. Brasília; 2013.

Empresa Brasileira de Pesquisa Agropecuária - Embrapa. Centro Nacional de Pesquisa de Solos. Manual de 338 métodos de análise de solos. $2^{\text {a }}$ ed. Rio de Janeiro; 2011.

Fox DM, Bryan RB, Fox CA. Changes in pore characteristics with depth for structural crusts. Geoderma. 2004;120:109-20.

Gennaro LA, Souza ZM, Weill MAM, Souza GS, Alves MC. Soil physical and microbiological attributes cultivated with the common bean under two management systems. R Ci Agron. 2014;45:641-9.

Hickmann C, Costa LM. Estoque de carbono no solo e agregados em Argissolo sob diferentes manejos de longa duração. R Bras Eng Agríc Amb. 2012;16:1055-61.

Hou XQ, Li R, Jia ZK, Han QF, Yang BP, Nie JF. Effects of rotational tillage practices on soil structure, organic carbon concentration and crop yields in semi-arid areas of northwest China. Soil Use Manage. 2012;28:551-8.

Juhász CEP, Cooper M, Cursi PR, Ketzer AO, Toma RS. Savanna woodland soil micromorphology related to water retention. Sci Agric. 2007;64:344-54.

Kemper WD, Chepil WS. Size distribution of aggregates. In: Black CA, editor. Methods of soil analysis. Madison: American Society of Agronomy; 1965. p.499-510.

Lima HV, Lima CLR, Leão TP, Cooper M, Silva AP, Romero RE. Tráfego de máquinas agrícolas e alterações de bioporos em área sob pomar de laranja. R Bras Ci Solo. 2005;29:677-84.

Mallory JJ, Mohtar RH, Heathman GC, Schulze DG, Braudeau E. Evaluating the effect of tillage on soil structural properties using the pedostructure concept. Geoderma. 2011;163:141-9.

Moreira FR, Dechen SCF, Silva AP, Figueiredo GC, De Maria IC, Pessoni PT. Intervalo hídrico ótimo em um Latossolo Vermelho cultivado em sistema semeadura direta por 25 anos. $\mathrm{R}$ Bras $\mathrm{Ci}$ Solo. 2014;38:118-27.

Pagliai M, Kutilek M. Soil micromorphology and soil hydraulics. New Trends Soil Micromorphol. 2008;1:5-19

Pagliai M, Vignozzi N, Pellegrini S. Soil structure and the effect of management practices. Soil Till Res. 2004;79:131-43.

Pires LF, Reichardt K, Cooper M, Cássaro FAM, Dias NMP, Bacchi OOS. Pore system changes of damaged Brazilian Oxisols and Nitosols induced by wet-dry cycles as seen in 2-D micromorphologic image analysis. An Acad Bras Ci. 2009;81:151-61.

Reichert JM, Suzuki LEAS, Reinert DJ. Compactação do solo em sistemas agropecuários e florestais: identificação, efeitos, limites críticos e mitigação. In: Ceretta CA, Silva LS, Reichert JM, editores. Tópicos em ciência do solo. Viçosa, MG: Sociedade Brasileira de Ciência do Solo; 2007. v.5, p.49-134.

Reynolds WD, Elrick DE. In situ measurement of field-saturated hydraulic conductivity, sorptivity, and the a-parameter using the Guelph permeameter. Soil Sci. 1985;140:292-302.

Rossetti KV, Centurion JF. Sistemas de manejo e atributos físico-hídricos de um Latossolo Vermelho cultivado com milho. R Bras Eng Agríc Amb. 2013;17:472-9.

Salton JC, Mielniczuk J, Bayer C, Boeni M, Conceição PC, Fabrício AC, Macedo MCM, Broch DL. Agregação e estabilidade de agregados do solo em sistemas agropecuários em Mato Grosso do Sul. R Bras Ci Solo. 2008;32:11-21.

Souza GS, Souza ZM, Silva RB, Barbosa RS, Araújo FS. Effects of traffic control on the soil physical quality and the cultivation of sugarcane. R Bras Ci Solo. 2014;38:135-46.

Souza ZM, Marques Júnior J, Cooper M, Pereira GT. Micromorfologia do solo e sua relação com atributos físicos e hídricos. Pesq Agropec Bras. 2006;41:487-92.

Vieira SR. Permeâmetro: novo aliado na avaliação de manejo do solo. Agronômico. 1998;47:32-3.

Williams SM, Weil RR. Crop cover root channels may alleviate soil compaction effects on soybean crop. Soil Sci Soc Am J. 2004;68:1403-9. 